\title{
Antipsychotic use and the risk of hip/femur fracture: a population-based case-control study
}

\author{
S. Pouwels • T. P. van Staa • A. C. G. Egberts • \\ H. G. M. Leufkens $\cdot$ C. Cooper $\cdot$ F. de Vries
}

Received: 30 September 2008 / Accepted: 4 December 2008 / Published online: 21 January 2009

(C) The Author(s) 2009. This article is published with open access at Springerlink.com

\begin{abstract}
Summary This case-control study showed that current use of conventional antipsychotics, but not atypical antipsychotics, seems to be associated with an increased risk of a hip/femur fracture, possibly related to the pharmacological properties of conventional antipsychotics. Furthermore, no evidence for a dose effect was found.

Introduction The aim of this study was to assess the risk of hip/femur fracture associated with antipsychotic use, with particular reference to any difference in risk with conventional versus atypical antipsychotics, dose, and pharmacological properties.

Methods A case-control study was conducted using data from the PHARMO Record Linkage System among individuals aged 18 years and older between 1991 and 2002 . Cases had a record of a hip or femur fracture, while controls had no evidence of ever having sustained any fracture.
\end{abstract}

S. Pouwels · T. P. van Staa • A. C. G. Egberts •

H. G. M. Leufkens $\cdot$ F. de Vries $(\bowtie)$

Department of Pharmacoepidemiology and Pharmacotherapy,

Utrecht Institute for Pharmaceutical Sciences, Utrecht University,

Utrecht, The Netherlands

e-mail: f.devries@uu.nl

T. P. van Staa $\cdot$ C. Cooper $\cdot$ F. de Vries

MRC Epidemiology Resource Centre,

University of Southampton, Southampton General Hospital,

Southampton, UK

A. C. G. Egberts

Department of Clinical Pharmacy,

University Medical Centre Utrecht,

Utrecht, The Netherlands

C. Cooper

Institute of Musculoskeletal Sciences, University of Oxford,

Oxford, UK
Results Most cases were elderly ( $77.6 \%$ aged $\geq 70$ years). We found an increased risk for hip/femur fracture associated with the use of antipsychotic drugs. The risk for current users $\left(\mathrm{OR}_{\mathrm{adj}} 1.68\right.$ [1.43, 1.99]) was significantly greater than with past use $\left(\mathrm{OR}_{\mathrm{adj}} 1.33[1.14,1.56] ; p=\right.$ 0.036). Current use of conventional antipsychotics $\left(\mathrm{OR}_{\mathrm{adj}}\right.$ $1.76[1.48,2.08])$ but not atypical antipsychotics $\left(\mathrm{OR}_{\mathrm{adj}}\right.$ $0.83[0.42,1.65])$ was associated with an increased risk. We did not find evidence for a dose effect.

Conclusion The use of conventional, but not atypical antipsychotics, seems to be associated with an increased risk of hip/femur fracture, possibly related to the pharmacological properties of conventional antipsychotics. However, the numbers of atypical antipsychotic users were small, and therefore this observation needs further attention in other study populations.

Keywords Antipsychotics · Bone density · Fracture .

Osteoporosis $\cdot$ Risk factors

\section{Introduction}

Antipsychotics are common in the treatment of schizophrenia, affective disorders, organic psychosis, and dementia [1, 2]. The side effects associated with antipsychotic use include sedation, extrapyramidal symptoms (EPS), and orthostatic hypertension, all of which may increase the risk of falls, especially during the initial period of exposure [3]. Conventional antipsychotics (e.g., haloperidol, chlorpromazine) and the atypical antipsychotic risperidone at high dose have a high affinity for dopamine $\mathrm{D}_{2}$ receptors [4]. This pharmacological property is clearly associated with the risk of EPS but also gives rise to elevated prolactin levels $[5,6]$. In contrast, most atypical antipsychotics like clozapine, olanzapine, 
quetiapine, and low-dose risperidone have a higher affinity for the 5-hydroxytryptamine-2A $\left(5-\mathrm{HT}_{2 \mathrm{~A}}\right)$ receptor than for dopamine $\mathrm{D}_{2}$ receptors [4]. Blocking of the 5- $\mathrm{HT}_{2 \mathrm{~A}}$ receptor has been associated with lowered prolactin levels. In contrary, the stimulating of $5-\mathrm{HT}_{2 \mathrm{~A}}$ receptors has been linked to increased prolactin levels [7]. The latter is the case when using a selective serotonin reuptake inhibitor (SSRI).

Elevated serum prolactin may reduce bone mineral density (BMD) in the long-term [6, 8, 9]. O'Keane and Meaney [10] found that the BMD of patients using prolactin-raising antipsychotics was significantly lower than that of users of antipsychotics without prolactin-raising properties. In line with these results are the findings that patients using SSRIs also experience a lower BMD [11] and have an increased risk of fracture [12].

Several epidemiological studies have reported an increased risk of hip or femur fracture among users of antipsychotics [13-19]. One study found a relationship between dose and use of antipsychotics, regardless of timing of exposure, although this was not reported for current users [17]. Liperoti et al. found no difference in fracture risk between conventional and atypical antipsychotics [15], whereas Howard et al. found an increased risk for individuals using prolactin-raising antipsychotics [13]. In addition, there is some evidence to suggest that men using antipsychotics have a greater risk of fracture than women [13].

The aims of this study were to evaluate the association between the use of antipsychotics and the risk of fracture of the hip or femur for men and women, to derive risk estimates separately for conventional and atypical antipsychotics, and to investigate the risk associated with dose and pharmacological properties.

\section{Methods}

Setting and study design

We conducted a case-control study within the Dutch PHARMO Record Linkage System (RLS) (www.pharmo.nl). The database includes the demographic details and complete medication histories for about one million community-dwelling residents in the Netherlands representing some $7 \%$ of the general population. Data are available from 1986 onwards and are linked to hospital discharge records as well as several other health registries, including pathology, clinical laboratory findings, and general practitioner data. Almost every individual in the Netherlands is registered with a single community pharmacy, independent of prescriber and irrespective of his or her health insurance or socioeconomic status. Pharmacy records have a high degree of completeness with regard to dispensed drugs [20]. Pharmacy data include information about the drug dispensed, the date of dispensing, the prescriber, the amount dispensed, the prescribed dosage regimen, and the estimated duration of use. Hospital discharge records include detailed information on date of admission, discharge diagnoses, and procedures. Validation studies on PHARMO RLS have confirmed a high level of data completeness and validity with regards to fractures [21]; PHARMO has been used more often to address risk factors of hip/femur fracture risk [22-24].

\section{Study population}

Data were collected for the period 1 January 1991 to 31 December 2002. Cases were patients aged 18 years and older with a record for a first fracture of the hip or femur during the study period. The date of hospital admission was used to define the index date. Each case was matched by year of birth, sex, and geographical region to up to four control patients without any evidence of ever having sustained a fracture during data collection. The controls were assigned the same index date as the corresponding case.

\section{Exposure assessment}

Exposure to antipsychotics (Anatomical and Therapeutic Chemical [ATC] category N05A excluding lithium [25]) was determined by reviewing dispensing information before the index date. "Current" users were patients who had been dispensed at least one antipsychotic within the 30-day period before the index date. "Recent" users were those who had been dispensed an antipsychotic between 31 and 182 days before the index date. "Past" users were patients who had one or more dispensings for an antipsychotic but who had stopped treatment more than 182 days before the index date.

For each current user, the average daily dose was estimated by dividing the total amount of antipsychotics dispensed by the treatment time. Average daily doses were expressed in haloperidol equivalents using defined daily dosages [25]. The duration of continuous use was calculated using the expected duration of use (in days) for each dispensing (the dispensed amount of the drug divided by the recorded dosage instruction). The total exposure period was defined as the sum of the total expected durations of use from all dispensings. If the period between two antipsychotic dispensings exceeded 6 months, this was considered a gap in treatment. Drugs dispensed before the gap were not included when calculating the period of continuous use.

Antipsychotic drugs were classified as atypical (quetiapine, clozapine, risperidone, olanzapine) or conventional (pipamperone, haloperidol, zuclopenthixol, thioridazine, levomepromazine, and "others"; Table 1). The most 
Table 1 Categorization of antipsychotic drugs and side effect profiles

\begin{tabular}{|c|c|c|c|c|c|}
\hline Group & Generic name properties & Sedative properties & EPS properties & Prolactin properties & $\mathrm{OH}$ \\
\hline \multirow[t]{6}{*}{ Atypical } & Clozapine & High & Low & Non-raising & High \\
\hline & Olanzapine & Medium & Low & Non-raising & Medium \\
\hline & Quetiapine & Medium & Low & Non-raising & Medium \\
\hline & Risperidone & Medium & Medium & - & Medium \\
\hline & Risperidone $\leq 4 \mathrm{mg} /$ day & - & - & Non-raising & - \\
\hline & Risperidone $>4 \mathrm{mg} /$ day & - & - & Raising & - \\
\hline \multirow[t]{5}{*}{ Conventional } & Haloperidol & Low & High & Raising & Low \\
\hline & Levomepromazine & High & Medium & Raising & Medium \\
\hline & Pipamperone & High & Low & Raising & Medium \\
\hline & Thioridazine & High & Low & Raising & High \\
\hline & Zuclopenthixol & Medium & Medium & Raising & High \\
\hline \multirow[t]{17}{*}{ Other conventional } & Benperidol & High & Low & Raising & Low \\
\hline & Bromperidol & Low & High & Raising & Low \\
\hline & Chlorpromazine & High & Medium & Raising & High \\
\hline & Chlorprothixene & Medium & Medium & Raising & High \\
\hline & Droperidol & Medium & Medium & Raising & Medium \\
\hline & Flupentixol & Low & Medium & Raising & Medium \\
\hline & Fluphenazine & Low & High & Raising & Medium \\
\hline & Fluspirilene & Low & Medium & Raising & Medium \\
\hline & Penfluridol & High & Medium & Raising & Low \\
\hline & Perazine & High & Low & Raising & High \\
\hline & Periciazine & High & Medium & Raising & Medium \\
\hline & Perphenazine & Medium & Medium & Raising & Low \\
\hline & Pimozide & Low & Medium & Raising & Low \\
\hline & Prochlorperazine & Medium & High & Raising & Medium \\
\hline & Sulpiride & Low & Medium & Raising & Low \\
\hline & Tiapride & Low & Low & Raising & Low \\
\hline & Trifluoperazine & Low & High & Raising & Low \\
\hline
\end{tabular}

$\mathrm{OH}$ orthostatic hypotension

recently dispensed antipsychotic was used to define the type. When more than one dispensing was issued, all dispensings were taken into account.

Among current users we assessed the sedative, extrapyramidal, prolactin-raising, and orthostatic hypotensive pharmacological properties of the antipsychotic dispensed as determined by an extensive review of the literature [1, 4, $6,26-32]$ (Table 1). If more than one antipsychotic had been prescribed before the index date, we selected the drug with the most severe side effect profile.

\section{Potential confounders}

The records of cases and controls were reviewed for evidence of potential confounders that have been associated with fracture risk $[33,34]$. These included a recent history (in the previous year) of anemia, mental disorders, impaired renal function, injuries, and skin or subcutaneous diseases and a history at any time of malignant neoplasm, endocrine disorder, cardiovascular disease, cerebrovascular disease, obstructive airway disease, inflammatory bowel disease, musculoskeletal or connective tissue disease, rheumatoid arthritis, polymyalgia rheumatica or ankylosing spondylitis. Other potential confounders included a dispensing within 3 months before the index date of a benzodiazepine or a prescription within the previous 6 months for any of the following: eye drops, bronchodilators, inhaled or oral corticosteroids, statins, hormone replacement therapy, lithium, antidepressants, beta-blockers, opioids, antiarrythmics, antiepileptics, thiazide diuretics, renin-angiotensin-aldosterone system (RAAS) inhibitors, thyroid and antithyroid hormones, drugs for diabetes, disease-modifying antirheumatic drugs (DMARDs), metoclopramide, $5 \mathrm{HT}_{3}$ antagonists, and two or more prescriptions for a non-steroidal antiinflammatory drug (NSAID).

\section{Statistical analysis}

Odds ratios (ORs) were derived for the risk of hip/femur fracture associated with the use of antipsychotics and the various potential confounding variables. Adjusted odds ratios $\left(\mathrm{OR}_{\mathrm{adj}}\right)$ for hip/femur fracture were estimated by comparing antipsychotic use with no use determined by conditional logistic regression analysis. Final regression 
Table 2 Characteristics of cases and controls

\begin{tabular}{lcr}
\hline Characteristic & $\begin{array}{c}\text { Cases (\%) } \\
(n=6,763)\end{array}$ & $\begin{array}{c}\text { Controls (\%) } \\
(n=26,341)\end{array}$ \\
\hline Age (years) & & \\
$18-49$ & $452(6.7)$ & $1,808(6.9)$ \\
$50-69$ & $1,061(15.7)$ & $4,239(16.1)$ \\
$\geq 70$ & $5,250(77.6)$ & $20,294(77.0)$ \\
Number of females & $4,929(72.9)$ & $19,138(72.7)$ \\
Medical history & & \\
Rheumatoid arthritis & $353(5.2)$ & $1,108(4.2)$ \\
Cardiovascular disease & $359(5.3)$ & $1,289(4.9)$ \\
Malignant neoplasm & $391(5.8)$ & $1,021(3.9)$ \\
Inflammatory bowel disease & $361(5.3)$ & $921(3.5)$ \\
Cerebrovascular disease & $296(4.4)$ & $565(2.1)$ \\
Drug use in 6 months before index date & \\
Oral glucocorticoids & $366(5.4)$ & $918(3.5)$ \\
DMARDs & $115(1.7)$ & $202(0.8)$ \\
Antidepressants & $643(9.5)$ & $1,343(5.1)$ \\
Anxiolytics & $1,170(17.3)$ & $3,451(13,1)$ \\
Antiepileptics & $494(7.3)$ & $938(3.6)$ \\
Lithium & $18(0.3)$ & $34(0.1)$ \\
Hormone replacement therapy & $77(1.1)$ & $347(1.3)$ \\
Bisphosphonates & $261(3.9)$ & $616(2.3)$ \\
\hline
\end{tabular}

models were determined by stepwise backward elimination using a significance level of 0.05 . Significant differences between categories were determined with the Wald statistic option of the PHREG procedure of SAS 9.1.
Analyses were conducted to evaluate the risk of fracture associated with current exposure to antipsychotics versus no use, grouping current users according to the daily dose of antipsychotic prescribed, whether the antipsychotic prescribed was conventional or atypical and according to the severity of expected side effects. We also stratified the study population to assess the risk with current use by age and sex.

\section{Results}

Table 2 shows the baseline characteristics of cases and controls. We identified 6,763 cases with a fracture of the hip or femur and 26,341 matched controls. Almost threequarters $(73 \%)$ of the study population was female. The mean duration of follow-up before the index date was 5.8 years for cases and 5.7 years for controls. The median age was 79 years for cases and controls. The median duration of use for current users was 30 days (determined from $94 \%$ of current users).

The use of antipsychotic drugs by cases and controls and the results of conditional logistic regression analysis are presented in Table 3. Antipsychotic drug use was significantly higher among cases compared with controls, with a trend towards increased risk of hip/femur fracture with recency of use. Current use of antipsychotics was associ-

Table 3 Risk of hip/femur fracture with antipsychotic use versus no use, including risk estimates (derived by conditional logistic regression analysis) for current use overall and by daily dose, and for current use by sex and age group

\begin{tabular}{|c|c|c|c|c|}
\hline Antipsychotic use $\mathrm{e}^{\mathrm{a}}$ & $\begin{array}{l}\text { Cases } \\
(n=6,763)\end{array}$ & $\begin{array}{l}\text { Controls } \\
(n=26,341)\end{array}$ & $\begin{array}{l}\text { Univariate analysis } \\
\text { OR }(95 \% \mathrm{CI})\end{array}$ & $\begin{array}{l}\text { Multivariate analysis } \\
\text { OR }(95 \% \mathrm{CI})\end{array}$ \\
\hline No use & 6,105 & 24,770 & Referent & Referent \\
\hline Past use & 249 & 653 & $1.57(1.35,1.83)$ & $1.33(1.14,1.56)^{\mathrm{c}}$ \\
\hline Recent use & 172 & 425 & $1.63(1.36,1.96)$ & $1.38(1.15,1.66)$ \\
\hline Current use & 237 & 493 & $2.00(1.70,2.35)$ & $1.68(1.43,1.99)^{\mathrm{c}}$ \\
\hline \multicolumn{5}{|c|}{ By average daily dose, $\mathrm{mg} /$ day $^{\mathrm{d}}$} \\
\hline First time users & 71 & 150 & $1.98(1.48,2.63)$ & $1.60(1.19,2.15)$ \\
\hline$<0.8$ & 60 & 122 & $2.04(1.49,2.79)$ & $1.79(1.30,2.47)$ \\
\hline $0.8-1.9$ & 60 & 126 & $2.01(1.47,2.75)$ & $1.66(1.20,2.30)$ \\
\hline$\geq 2$ & 46 & 95 & $1.96(1.37,2.80)$ & $1.71(1.19,2.46)$ \\
\hline \multicolumn{5}{|l|}{ By gender } \\
\hline Females & 193 & 419 & $1.90(1.59,2.27)$ & $1.63(1.36,1.96)$ \\
\hline Males & 44 & 74 & $2.53(1.72,3.72)$ & $1.93(1.28,2.90)$ \\
\hline \multicolumn{5}{|l|}{ By age category } \\
\hline Ages $18-69$ years & 15 & 35 & $1.78(0.97,3.28)$ & $0.95(0.48,1.87)$ \\
\hline Ages $\geq 70$ years & 222 & 458 & $2.00(1.69,2.37)$ & $1.74(1.46,2.06)$ \\
\hline
\end{tabular}

${ }^{\text {a }}$ For current, recent, and past users, the last antipsychotic was dispensed respectively within 30 days, between 31 and 182 days, and more than 182 days prior to the index date

${ }^{\mathrm{b}}$ Adjusted for a history of malignant neoplasm, anemia, endocrine disorders, skin or subcutaneous disease, cerebrovascular disease, obstructive airway disease, musculoskeletal or connective tissue disease, use of benzodiazepines, inhaled or oral glucocorticoids, statins, antidepressants, beta-blockers, opioids, antiepileptics, RAAS inhibitors, drugs for diabetics, DMARDs, metoclopramide, and two or more NSAID dispensing ${ }^{\mathrm{c}}$ Significant difference between current and past use of antipsychotics ( $p=0.036$ after Wald test)

${ }^{\mathrm{d}}$ Haloperidol equivalents 


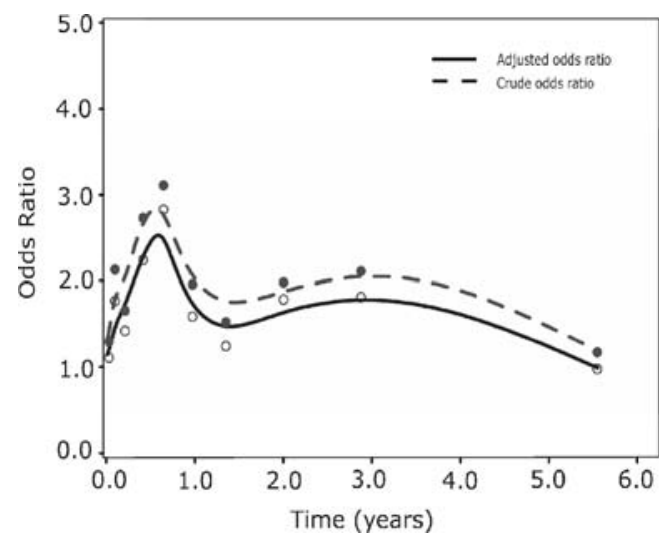

Fig. 1 The risk of hip/femur fracture with duration of continuous antipsychotic use (years) before the index date among current users

ated with a significantly increased risk of hip/femur fracture compared with no use ( $\mathrm{OR}_{\text {adj }} 1.68$ [95\% CI 1.43, 1.99]) and the risk associated with current use was significantly greater than that associated with past use $\left(\mathrm{OR}_{\mathrm{adj}} 1.33[95 \% \mathrm{CI}\right.$ $1.14,1.56] ; p=0.036$ ). When current use was defined by daily dose, the risk estimates for fracture did not demonstrate a dose-response relationship. Further stratified analyses suggested that the risk of hip/femur fracture for current users of antipsychotics was greater for men $\left(\mathrm{OR}_{\mathrm{adj}}\right.$ $1.93[95 \% \mathrm{CI} 1.28,2.90])$ than for women $\left(\mathrm{OR}_{\mathrm{adj}} 1.63\right.$ [95\% CI 1.36, 1.96]), although not significantly so. Similarly, risk was increased for individuals aged $\geq 70$ years $\left(\mathrm{OR}_{\text {adj }} 1.74\right.$ [95\% CI 1.46, 2.06]), but not for younger patients $\left(\mathrm{OR}_{\text {adj }} 0.95\right.$ [95\% CI $\left.\left.0.48,1.87\right]\right)$.
Figure 1 presents ORs for hip/femur fracture with duration of continuous use before the index date among current users. There was a marked increase in fracture risk during the first 8 months of continuous antipsychotic use $\left(\mathrm{OR}_{\mathrm{adj}} 2.83\right.$ [95\% CI 1.75, 4.57]) and evidence to suggest a second period of increased risk as the duration of continuous use approached 2 years.

The current use of atypical antipsychotics did not appear to increase the risk of hip/femur fracture $\left(\mathrm{OR}_{\text {adj }} 0.83[95 \% \mathrm{CI}\right.$ $0.42,1.65]$; Table 4). The risk associated with current use of conventional antipsychotics $\left(\mathrm{OR}_{\text {adj }} 1.76\right.$ [95\% CI 1.48 , $2.08])$ was increased, however, and was significantly greater than with the use of atypical antipsychotics $(p=0.038)$.

Table 5 presents the ORs for hip/femur fracture according to the pharmacological profile of the antipsychotic in current use. The use of antipsychotics with high prolactinraising properties (i.e., most conventional antipsychotics and risperidone $>4 \mathrm{mg}$ /day) was associated with an increased risk of hip/femur fracture $\left(\mathrm{OR}_{\mathrm{adj}} 1.75[95 \% \mathrm{CI}\right.$ $1.48,2.08]$ ), whereas antipsychotics with low prolactinraising properties (i.e., most atypical antipsychotics including risperidone $\leq 4 \mathrm{mg} /$ day) were not associated with an increased risk of fracture $\left(\mathrm{OR}_{\mathrm{adj}} 0.91\right.$ [95\% CI 0.45, 1.85)]. After comparison of both groups, no significant difference was observed. Analysis stratifying current use according to the EPS properties of the antipsychotics suggested a trend towards increased risk with increasing EPS $\left(\mathrm{OR}_{\mathrm{adj}} 1.55\right.$ $[95 \%$ CI $1.18,2.04]$ for low EPS and $\mathrm{OR}_{\text {adj }} 1.97[95 \% \mathrm{CI}$ $1.49,2.61]$ for high EPS), but this trend did not reach statistical significance. There was no apparent association

Table 4 Risk of hip/femur fracture with current antipsychotic use according to class and type of antipsychotic

\begin{tabular}{|c|c|c|c|c|}
\hline Antipsychotic use ${ }^{a}$ & $\begin{array}{l}\text { Cases } \\
(n=6,763)\end{array}$ & $\begin{array}{l}\text { Controls } \\
(n=26,341)\end{array}$ & $\begin{array}{l}\text { Univariate analysis } \\
\text { OR }(95 \% \mathrm{CI})\end{array}$ & $\begin{array}{l}\text { Multivariate analysis } \\
\text { OR }(95 \% \text { CI })\end{array}$ \\
\hline No use & 6,105 & 24,770 & Referent & Referent \\
\hline Past use & 249 & 653 & $1.57(1.35,1.83)$ & $1.33(1.14,1.56)$ \\
\hline Recent use & 172 & 425 & $1.63(1.36,1.96)$ & $1.38(1.15,1.66)$ \\
\hline Current use & 237 & 493 & $2.00(1.70,2.35)$ & $1.68(1.43,1.99)$ \\
\hline Conventional antipsychotics ${ }^{\mathrm{c}}$ & 227 & 453 & $2.08(0.48,1.86)$ & $1.76(1.48,2.08)^{\mathrm{d}}$ \\
\hline Pipamperone & 70 & 165 & $1.71(1.29,2.28)$ & $1.54(1.15,2.06)$ \\
\hline Haloperidol & 75 & 106 & $2.87(2.13,3.86)$ & $2.33(1.72,3.18)$ \\
\hline Zuclopenthixol & 38 & 56 & $2.78(1.83,4.21)$ & $2.44(1.59,3.75)$ \\
\hline Thioridazine & 7 & 17 & $1.59(0.64,3.93)$ & $1.51(0.60,3.78)$ \\
\hline Levomepromazine & 8 & 27 & $1.01(0.45,2.28)$ & $0.80(0.35,1.82)$ \\
\hline Others & 34 & 96 & $1.39(0.93,2.07)$ & $1.19(0.79,1.78)$ \\
\hline Atypical antipsychotics ${ }^{\mathrm{c}}$ & 11 & 44 & $0.95(0.48,1.86)$ & $0.83(0.42,1.65)^{\mathrm{d}}$ \\
\hline Risperidone & 8 & 32 & $0.95(0.43,2.10)$ & $0.84(0.38,1.88)$ \\
\hline Quetiapine, olanzapine, clozapine & 3 & 12 & $0.93(0.26,3.34)$ & $0.83(0.23,3.02)$ \\
\hline
\end{tabular}

${ }^{a}$ If more than one antipsychotic had been dispensed before the index date, then all dispensings were taken into account. For current, recent, and past users, the last antipsychotic was dispensed respectively within 30 days, between 31 and 182 days, and more than 182 days prior to the index date

${ }^{\mathrm{b}}$ Adjusted for confounders as presented in Table 3

${ }^{\mathrm{c}}$ In both the univariate as is the multivariate analysis also adjusted for other antipsychotics

${ }^{\mathrm{d}}$ Significant difference between conventional antipsychotics and atypical antipsychotics ( $p=0.038$ after Wald test). 
Table 5 Risk of hip/femur fracture with current antipsychotic use according to the pharmacological properties

\begin{tabular}{|c|c|c|c|c|}
\hline Antipsychotic use ${ }^{a}$ & $\begin{array}{l}\text { Cases } \\
(n=6,763)\end{array}$ & $\begin{array}{l}\text { Controls } \\
(n=26,341)\end{array}$ & $\begin{array}{l}\text { Univariate analysis } \\
\text { OR }(95 \% \mathrm{CI})\end{array}$ & $\begin{array}{l}\text { Multivariate analysis } \\
\text { OR }(95 \% \mathrm{CI})\end{array}$ \\
\hline No use & 6,105 & 24,770 & Referent & Referent \\
\hline Past use & 249 & 653 & $1.57(1.35,1.83)$ & $1.33(1.14,1.56)$ \\
\hline Recent use & 172 & 425 & $1.63(1.36,1.96)$ & $1.38(1.15,1.66)$ \\
\hline Current use & 237 & 493 & $2.00(1.70,2.35)$ & $1.68(1.43,1.99)$ \\
\hline \multicolumn{5}{|l|}{ Sedative properties } \\
\hline Low & 89 & 144 & $2.54(1.95,3.31)$ & $2.09(1.59,2.74)$ \\
\hline Medium & 53 & 125 & $1.78(1.28,2.47)$ & $1.50(1.07,2.10)$ \\
\hline High & 95 & 224 & $1.75(1.37,2.24)$ & $1.51(1.17,1.94)$ \\
\hline \multicolumn{5}{|l|}{ EPS properties } \\
\hline Low & 80 & 191 & $1.73(1.33,2.26)$ & $1.55(1.18,2.04)$ \\
\hline Medium & 74 & 163 & $1.90(1.44,2.51)$ & $1.58(1.18,2.10)$ \\
\hline High & 83 & 139 & $2.46(1.87,3.24)$ & $1.97(1.49,2.61)$ \\
\hline \multicolumn{5}{|l|}{ Prolactin properties } \\
\hline Non-raising & 10 & 39 & $1.06(0.52,2.12)$ & $0.91(0.45,1.85)$ \\
\hline Raising & 227 & 454 & $2.08(1.76,2.45)$ & $1.75(1.48,2.08)$ \\
\hline \multicolumn{5}{|c|}{ Orthostatic hypotensive properties } \\
\hline Low & 97 & 157 & $2.55(1.98,3.29)$ & $2.08(1.60,2.71)$ \\
\hline Medium & 92 & 257 & $1.49(1.17,1.90)$ & $1.27(0.99,1.64)$ \\
\hline High & 48 & 79 & $2.50(1.74,3.59)$ & $2.19(1.51,3.18)$ \\
\hline
\end{tabular}

${ }^{a}$ When more than one antipsychotic was dispensed simultaneously before the index date, then the antipsychotic with the most severe side effect was selected. For current, recent, and past users, the last antipsychotic was dispensed respectively within 30 days, between 31 and 182 days, and more than 182 days prior to the index date

${ }^{\mathrm{b}}$ Adjusted for confounders as before

between the degree of potential orthostatic hypotensive or sedative side effects and the risk of hip/femur fracture.

\section{Discussion}

The findings of this study have demonstrated an increased risk of hip/femur fracture with the use of antipsychotics. The risk was highest for current users, especially the most elderly. The use of conventional antipsychotics appeared to account for the increased risk, and there was evidence for an increased risk with prolactin-raising antipsychotics and those with greater potential to affect the extrapyramidal system. We did not find evidence to support an association between the average daily dose of antipsychotic and the risk of hip/femur fracture.

Our findings confirm an association described in other epidemiological studies on the risk of hip/femur fracture with the use of antipsychotics [13-19]. The 1.7-fold increased risk of fracture among current users and declining risk after discontinuation of use agrees with the findings of others. Hugenholtz et al. [18] reported a 1.3-fold increased adjusted risk of fracture among current users who had been using antipsychotics long term, and produced a plot similar to ours for risk with cumulative days of treatment (Fig. 1). Ray et al. [16] reported a doubling of risk among current users (OR $2.0[95 \%$ CI 1.6, 2.6]), although that risk estimate may have been reduced with adjustment for more potential confounding variables.

In agreement with other recent studies, we did not find an association between the average daily dose of antipsychotic and the risk of hip/femur fracture for current users $[17,18]$. Vestergaard et al. [17] described a dose-response relationship for all users of antipsychotics before the index date but the association was not apparent for current users and the elapsed time between the last dispensing and the index date could have been as much as 4 years. Although we found a higher fracture risk for men currently using antipsychotics, the difference between the sexes was not significant. A greater fracture risk for men using antipsychotics has been reported before [13], however, which could reflect the effects of antipsychotic use and physiological processes promoting bone loss [9].

The association between the risk of hip/femur fracture and the EPS and prolactin-raising properties of the antipsychotic prescribed could explain the shape of curve derived by plotting the OR for fracture risk against the duration of antipsychotic use (Fig. 1). The symptoms associated with extrapyramidal effects often start soon after the initiation of treatment and may be transient [35]. In addition, the sedative and orthostatic hypotensive side effects of antipsychotics often occur immediately after the start of treatment. The second period of increased risk after several months of use may reflect the effects of long-term 
hyperprolactinemia on bone density. Indeed, Hugenholtz et al. [20] found an increased risk only among long-term users of antipsychotics and attributed this to the prolactin-raising properties of antipsychotics. We did not find an association between the sedative and orthostatic hypotensive side effects and fracture risk in our analyses.

One of the strengths of our study is the size of the study population (6,763 cases and 26,341 controls) and that it is representative for the general population of the Netherlands, although the absolute number of users of atypical antipsychotics was low. All prescribing information was collected routinely and we do not expect our findings to be biased with regards to exposure status. Also, as fractures invariably result in hospitalization, we are confident that cases, controls, and index dates were identified reliably. Nevertheless, given the observational nature of this study, the results should be interpreted with knowledge of its limitations. First, cases and controls were not matched on the period of observation available in the database and the results could be affected by information bias. However, the exclusion of patients with less than 1 year of follow-up did not affect the results substantially. Second, information about relevant diagnoses and co-morbidities may have been recorded upon hospitalization for a fracture and it is likely that the information available for cases was more complete and up-to-date than that available for controls. It could be argued that we did not consider the use of bisphosphonates as a potential confounder. However, there should be a priori evidence, that a confounder is associated both with antipsychotic exposure and hip fracture risk. As far as we know, there is no clear evidence that antipsychotic users are more likely to be exposed to bisphosphonates, compared to non-users. Moreover, in a case-control study, the use of bisphosphonates may act as an intermediate variable between exposure and outcome, rather than a confounder. This is supported by the positive association between bisphosphonate use and hip fracture (crude OR 1.71 [95\% CI 1.47, 1.99], Table 2). Another potential limitation is the unavailability of data on smoking and alcohol consumption for a population that may include individuals with high levels of nicotine and/or alcohol consumption. Both are well-known risk factors of fracture risk [36, 37]. The possibility remains, therefore, that missing data on alcohol and smoking habit could (partially) explain the positive association between antipsychotic use and fracture risk.

Finally, the comparison between conventional and atypical antipsychotics should be interpreted with caution, because the analyses in the group of atypical antipsychotic users are based on a limited number of patients. Furthermore, atypical antipsychotics were introduced later into clinical use than typical antipsychotics, which may have led to different fracture risk profiles. Further studies are required to confirm these results. The same applies for the results regarding the prolactin-raising properties.

Confounding by indication is an alternative explanation for the observed association between use of antipsychotics and risk of hip fracture. The PHARMO database does not contain routinely collected information on, for example, cognitive disorders and mental illnesses for the majority of their patients. Schizophrenia has been associated with perturbations in bone metabolism [10]. However, a study among $>3,600$ Finnish institutionalized elderly (mean age 83 years) showed that only $4 \%$ were diagnosed with schizophrenia, whereas $58 \%$ suffered from dementia, and $16 \%$ suffered from depression. A substantial number $(41 \%)$ of patients with dementia or depression were prescribed antipsychotics. Furthermore, of $11-30 \%$ of all patients who had behavioral problems such as wandering, being physically or verbally abusive, or who resisted care, $48-64 \%$ were prescribed an antipsychotic at least once a year [38]. Jeste et al. confirmed that antipsychotics are often prescribed off-label for behavioral disturbances associated with dementia [39]. Because dementia $[40,41]$ and depression [42] are risk factors for fractures, they may be an alternative explanation for the positive association between antipsychotic use and risk of hip/femur fracture. This hypothesis is in line with the findings of Bolton et al. who investigated antipsychotic use and the risk of fractures, but found no increased risk among both conventional and atypical antipsychotic users. In this study, the results were adjusted for a wide range of confounders including dementia, schizophrenia, and depression [43].

In conclusion, our findings support an increased risk for fracture of the hip or femur for individuals prescribed antipsychotics. There was a difference in fracture risk with the use of atypical versus conventional antipsychotics, wherein patients using conventional antipsychotic drugs had an increased risk of hip/femur fracture. However, it should be noted that the numbers of atypical antipsychotic users were small, and that this observation needs further attention in other study populations. We did not find a relationship between average daily dose of antipsychotic and fracture risk. While the possibility remains that the underlying disease or behavior caused any increased risk of hip/femur fractures, our findings may provide important information for prescribers, especially those managing elderly and vulnerable patients.

Acknowledgments The authors would like to thank Dr Helen Seaman for her assistance in the preparation of this manuscript for publication. The study was not funded.

Conflicts of interest The Department of Pharmacoepidemiology and Pharmacotherapy employing authors S. Pouwels, T.P. van Staa, A.C.G. Egberts, H.G.M. Leufkens and F. de Vries have received unrestricted funding for pharmacoepidemiological research from GlaxoSmithKline, Novo Nordisk, the private-public funded Top Institute Pharma (www. tipharma.nl, includes co-funding from universities, government, and industry), the Dutch Medicines Evaluation Board, and the Dutch 
Ministry of Health. Dr. van Staa and Dr. de Vries also work for the General Practice Research Database (GPRD), UK. GPRD is owned by the UK Department of Health and operates within the Medicines and Healthcare products Regulatory Agency (MHRA). GPRD is funded by the MHRA, Medical Research Council, various universities, contract research organizations, and pharmaceutical companies.

Open Access This article is distributed under the terms of the Creative Commons Attribution Noncommercial License which permits any noncommercial use, distribution, and reproduction in any medium, provided the original author(s) and source are credited.

\section{References}

1. Rang HP et al (1999) Pharmacology, 4th edn. Churchill Livingstone, Edinburgh

2. Jeste DV, Dolder CR (2004) Treatment of non-schizophrenic disorders: focus on atypical antipsychotics. J Psychiatr Res 38 (1):73-103

3. Neutel CI, Perry S, Maxwell C (2002) Medication use and risk of falls. Pharmacoepidemiol Drug Saf 11(2):97-104

4. Miyamoto S et al (2005) Treatments for schizophrenia: a critical review of pharmacology and mechanisms of action of antipsychotic drugs. Mol Psychiatry 10(1):79-104

5. Melkersson KI, Hulting AL, Rane AJ (2001) Dose requirement and prolactin elevation of antipsychotics in male and female patients with schizophrenia or related psychoses. $\mathrm{Br} \mathrm{J}$ Clin Pharmacol 51(4):317-324

6. Haddad PM, Wieck A (2004) Antipsychotic-induced hyperprolactinaemia: mechanisms, clinical features and management. Drugs 64(20):2291-314

7. Van de Kar LD et al (2001) 5-HT2A receptors stimulate ACTH, corticosterone, oxytocin, renin, and prolactin release and activate hypothalamic CRF and oxytocin-expressing cells. J Neurosci 21 (10):3572-3579

8. Misra M, Papakostas GI, Klibanski A (2004) Effects of psychiatric disorders and psychotropic medications on prolactin and bone metabolism. J Clin Psychiatry 65(12):1607-1618 quiz $1590,1760-1761$

9. Meaney AM et al (2004) Effects of long-term prolactin-raising antipsychotic medication on bone mineral density in patients with schizophrenia. Br J Psychiatry 184:503-508

10. O'Keane V, Meaney AM (2005) Antipsychotic drugs: a new risk factor for osteoporosis in young women with schizophrenia. J Clin Psychopharmacol 25(1):26-31

11. Diem SJ et al (2007) Use of antidepressants and rates of hip bone loss in older women: the study of osteoporotic fractures. Arch Intern Med 167(12):1240-1245

12. Richards JB et al (2007) Effect of selective serotonin reuptake inhibitors on the risk of fracture. Arch Intern Med 167(2):188-194

13. Howard L, Kirkwood G, Leese M (2007) Risk of hip fracture in patients with a history of schizophrenia. Br J Psychiatry 190:129-134

14. Cumming RG, Klineberg RJ (1993) Psychotropics, thiazide diuretics and hip fractures in the elderly. Med J Aust 158 (6):414-417

15. Liperoti $\mathrm{R}$ et al (2007) Conventional or atypical antipsychotics and the risk of femur fracture among elderly patients: results of a case-control study. J Clin Psychiatry 68(6):929-934

16. Ray WA et al (1987) Psychotropic drug use and the risk of hip fracture. N Engl J Med 316(7):363-369

17. Vestergaard P, Rejnmark L, Mosekilde L (2006) Anxiolytics, sedatives, antidepressants, neuroleptics and the risk of fracture. Osteoporos Int 17(6):807-816
18. Hugenholtz GW et al (2005) Risk of hip/femur fractures in patients using antipsychotics. Bone 37(6):864-870

19. Sernbo I, Hansson A, Johnell O (1987) Drug consumption in patients with hip fractures compared with controls. Compr Gerontol [A] 1(3):93-96

20. Buurma $\mathrm{H}$ et al (2008) Prevalence and determinants of pharmacy shopping behaviour. J Clin Pharm Ther 33(1):17-23

21. Herings RM et al (1996) Current use of thiazide diuretics and prevention of femur fractures. J Clin Epidemiol 49(1):115-119

22. de Vries F et al (2007) Use of inhaled and oral glucocorticoids, severity of inflammatory disease and risk of hip/femur fracture: a population-based case-control study. J Intern Med 261(2):170-177

23. de Vries F et al (2007) Use of beta-2 agonists and risk of hip/ femur fracture: a population-based case-control study. Pharmacoepidemiol Drug Saf 16(6):612-619

24. de Vries F et al (2007) Use of beta-blockers and the risk of hip/ femur fracture in the United Kingdom and The Netherlands. Calcif Tissue Int 80(2):69-75

25. WHO (2005) WHO Collaborating Centre for drug statistics methodology. The ATC/DDD system. World Health Organisation

26. Becker D et al (2003) Risperidone, but not olanzapine, decreases bone mineral density in female premenopausal schizophrenia patients. J Clin Psychiatry 64(7):761-766

27. Koda-Kimble MA, Young LY, Kradjan WA (2003) Applied therapeutics: the clinical use of drugs, 7th edn. . Lippincott, Williams \& Wilkins, New York

28. Speight TM, Holford NHG (1997) Avery's drug treatment: A guide to the properties, choice, therapeutic use and economic value of drugs in disease management, 4th edn. Adis Press, Auckland

29. AMAM (1996) American Medical Association. Division of Drugs and Toxicology. Drug Evaluations Annual, Chicago

30. Hummer M et al (2005) Osteoporosis in patients with schizophrenia. Am J Psychiatry 162(1):162-167

31. Petty RG (1999) Prolactin and antipsychotic medications: mechanism of action. Schizophr Res 35(Suppl):S67-S73

32. Warrel DA, Cox TM, Firth JD (2005) Oxford textbook of medicine, vol. 3. 4th edn. Oxford University Press, Oxford

33. Grisso JA, Capezuti E, Schwartz A (1996) Falls as risk factors for fractures. In: Marcus D, Kelsey J, Feldman D (eds) Osteoporosis. Academic, San Diego, pp 599-611

34. Cummings SR et al (1995) Risk factors for hip fracture in white women. Study of osteoporotic fractures research group. N Engl J Med 332(12):767-773

35. Owens DC (1999) A guide to the extrapyramidal side-effects of antipsychotic drugs. Cambridge University Press, Cambridge

36. Kanis JA et al (2005) Smoking and fracture risk: a meta-analysis. Osteoporos Int 16(2):155-162

37. Cauley JA et al (2005) Factors associated with the lumbar spine and proximal femur bone mineral density in older men. Osteoporos Int 16(12):1525-1537

38. Alanen HM et al (2006) Use of antipsychotic medications among elderly residents in long-term institutional care: a three-year follow-up. Int J Geriatr Psychiatry 21(3):288-295

39. Jeste DV et al (2008) ACNP white paper: update on use of antipsychotic drugs in elderly persons with dementia. Neuropsychopharmacology 33(5):957-970

40. Melton LJ III et al (1994) Fracture risk in patients with Alzheimer's disease. J Am Geriatr Soc 42:614-619

41. van Staa TP et al (2002) Utility of medical and drug history in fracture risk prediction among men and women. Bone 31:508-514

42. Whooley MA et al (1999) Depression, falls, and risk of fracture in older women. Arch Intern Med 159(5):484-490

43. Bolton JM et al (2008) Fracture risk from psychotropic medications: a population-based analysis. J Clin Psychopharmacol 28 (4):384-391 